\title{
When Parents Need Not Have Children -- Cognitive Biases in Information Modeling
}

\author{
Keng Siau, Yair Wand, Izak Benbasat \\ Faculty of Commerce \& Business Administration \\ The University of British Columbia \\ 2053 Main Mall , Vancouver, B.C. \\ Canada V6T 172
}

\begin{abstract}
Cognitive biases associated with human judgment and choice are widely studied, recognized, and documented in behavioral decision research. It is also well accepted that an understanding and acknowledgment of these biases are vital to mitigate their effects. However, research into cognitive biases in information modeling is virtually lacking. Lest one assumes that research on cognitive biases is irrelevant to the field, information modeling is a cognitively intensive activity and is, thus, highly susceptible to such biases. There is a pressing need then, to identify and understand these human biases in order to lessen their effects. This paper describes an experiment designed to investigate the use of syntactic and semantic information by modeling experts. The experimental results indicate that when interpreting information models, modeling experts tend to focus on the syntactic aspects of information and totally ignore the semantic information, even in situations where the semantic information is clearly more representative of the real world situation. These biases exhibited by modeling experts are explained using the learning paradigm in cognitive psychology.
\end{abstract}

\subsection{Introduction}

An expert is one who does not have to think. He knows.

-- Frank Lloyd Wright

Information modeling can be defined as "the activity of formally describing some aspects of the physical and social world around us for the purpose of understanding and communication" (Mylopoulos 1992). Information modeling involves investigating the problems and requirements of the user community, and from that, building a requirements specification for the desired system (Rolland \& Cauvet 1992, Kangassalo 1990). The product of the information modeling process is an information model which serves as the link between requirements specification and systems development. 
The information model plays two important roles. First, it provides the basis for communicating and thinking about information systems during the analysis phase (Willumsen 1993). Second, it serves as the foundation for tools and techniques employed in the development of information systems (Kung \& Solvberg 1986). The importance of a correct information model cannot be overemphasized. Boehm (1976) made this point clearly when he stated:

\section{It is estimated that the relative cost of fixing problems detected during final testing or operation is 50-100 times greater than for problems detected during requirements specification.}

To achieve accurate information systems requirements, the analysts and end users must understand and communicate with one another effectively (Holtzblott \& Beyer 1995). However, information requirements specification is no easy task. Brooks $(1987, p$. 16) writes "the hardest part of the software task is arriving at a complete and consistent specification." Gladden (1982) estimated that information systems failures run as high as $70 \%$ of all information systems projects and Boar (1984) reported that $60-80 \%$ of these failures result from poor requirements specification. Also, research (e.g., Lientz \& Swanson 1980, Ramamoorthy et al. 1984) has shown that about twothirds of the maintenance cost can be attributed to misconception -- not identifying the real needs, or improper conceptual design.

Misunderstanding and miscommunication result from the different views of the analysts and end users. Because of the differences in background and training, the analysts and end users have very different perspectives. End users are experts in the application domain, but are usually untrained in the application of modeling techniques. It is plausible that, therefore, they will tend to focus on the semantic information depicted in the information models. Analysts, on the other hand, are experts in the modeling techniques, but unaware of the requirements of the organizations. Thus, it is likely that they will pay more attention to syntactic information in the models and less to the semantic information. To use the "blind men and the elephant" analogy, the one who felt the elephant's tail had quite a different view of the elephant from the one who felt its trunk. Therefore, the analysts and end users, during the information modeling phase, might be looking at the same information models, but reach different conclusions eventually. For example, the study by Curtis et al. (1988) showed that the modeler may became trapped in initial representations of the information model and failed to see alternative representations. Such biases are collectively known as cognitive biases (Tversky \& Kahneman, 1982)

Because information modeling is a cognitively intensive activity, such an activity is susceptible to cognitive biases. Thus, there is a need for us to identify and understand these sources of biases in order to recognize and control them. Researchers such as Stacy (1995), and Stacy and MacMillian (1995) have also argued for the importance of understanding cognitive biases in software development. They argued that the 
awareness of these human biases will lead to better software engineering practice, and better understanding between the analysts and end-users.

\subsection{Cognitive Biases}

Cognitive biases associated with human judgment and choice are extensively researched in behavioral decision making arena (see Baron, 1988, Hogarth, 1980; Tversky \& Kahneman, 1982). It is widely acknowledged that these biases can interfere with decision making processes and affect the quality of the decisions. The majority of these biases arise as a result of limitations in human information processing capability (Newell \& Simon, 1972). Despite the acceptance and recognition of cognitive biases in human decision making, few researchers of information modeling have attempted to investigate the effect of cognitive biases in information modeling. Information modeling, nonetheless, is susceptible to cognitive biases of analysts and end-users because models are abstractions of the real-world (Holland et al. 1986). In constructing and interpreting information models, analysts and end-users are likely to use their own common sense knowledge, domain knowledge, heuristics, and rules-of-thumb to assist them in their tasks. Such personalized knowledge and heuristic are highly subjective and may be detrimental to the understanding and communication aspects of information models which are the fundamental goals of information modeling.

\subsection{Outline of the Paper}

In this paper, we report an experimental study which clearly demonstrates the biases of modeling experts when they are given contradictory syntactic and semantic information in information modeling. The findings are discussed and the theoretical explanations from cognitive psychology suggested.

\subsection{Research Framework}

\subsection{Research Question}

Two types of information are represented in any modeling technique: syntactic and semantic information. Syntactic information is defined as the order of language units, as specified by the grammatical rules of the modeling techniques (Mayer 1983). Semantics information, on the other hand, refers to the meaning or referents represented in the information model. As an example, consider the information model given below. 


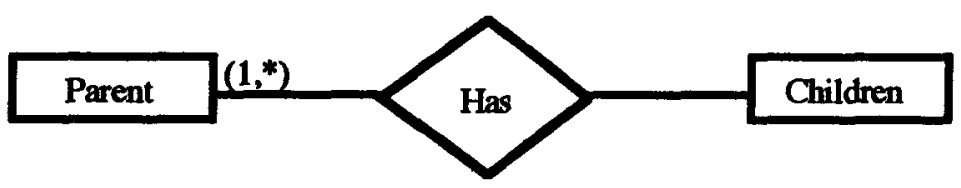

\section{An Information Model}

The rules and notations governing the use and interpretation of entities, relationships, and structural constraints (i.e., $(1, *))$ in the model represent the syntactic information. On the other hand, the idea that "parents have children" is the semantic information.

In our previous study (Siau et al. 1995), we investigated the use of relationship concept by modeling experts. The results show a predominant use of optional over mandatory relationships by expert users for both familiar and unfamiliar domains. This raises the question of whether modeling experts pay attention to the semantic information depicted by the information models.

The objective of this research is to extend our precious study and analyze the use of syntactic and semantic information by modeling experts. The main difficulty in this study is to separate the syntactic from the semantic information. Without a clear separation, the research question could not be investigated because of the confounding effect of one type of information on the other. How could we separate the syntactic from the semantic information?

One good way to differentiate the two is to introduce a conflict in the syntactic and semantic information. For example, we could use the idea "Parents have children" and then introduce a conflict into the information model by presenting a syntactic information that "Parents need not have children." In other words, the semantic information and the syntactic information are now in contradiction. This design enables us to investigate the use of the two types of information by the subjects.

The conflicting syntactic information is manipulated using structural constraints. Structural constraint is defined as the cardinality ratio and participation constraints taken together (Elmasri \& Navathe 1994). It is represented by a pair of integer numbers ( $\min , \max )$ with each participation of an entity type $E$ in a relationship type $R$, where $0 \leq \min \leq \max$ and $\max \geq 0$. The numbers mean that for each entity $e$ in $E, e$ must participate in at least min and at most max relationship instances in $R$ at all time.

Broadly speaking, structural constraints can be classified into two categories: optional and mandatory. If the minimum participation is 0 , it is an optional relationship and if the minimum participation is greater than 0 , it is a mandatory relationship. In our study, the mandatory constraint is depicted using the notation $(1, *)$ whilst the optional constraint is shown using the notation $(0, *)$. The structural constraints can be used to create a conflicting information model. For example, the first model below 
is conflicting since the semantic information tells us that parent must have children (i.e., knowledge from real world) but the syntactic information (i.e., depicted by the structural constraint -- $(0, *)$ ) suggests an optional relationship. The second diagram, however, is non-conflicting because the conflicting structural constraint is absent.

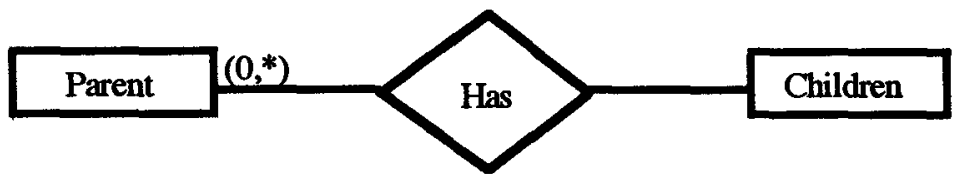

\section{Conflicting Model}

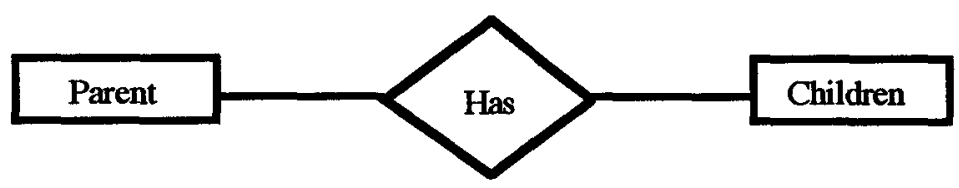

\section{Non-Conflicting Model}

The experiment consists of two groups of subjects. The first group is exposed to information models that have a conflict between the syntactic and semantic information. The second group is exposed to information models that do not have such a conflict. The research framework for the study reported in this paper is summarized below. ${ }^{1}$

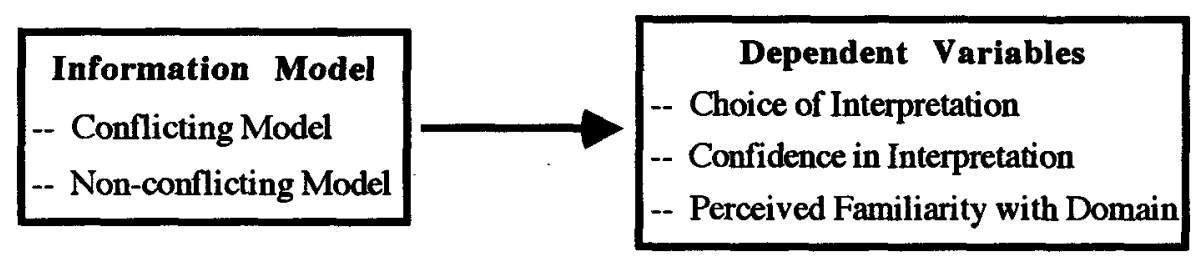

\section{Experimental Framework}

1 The entire design of the experiment consists of 3 cells. The first cell consists of models with conflicting syntactic and semantic information. The models in the second cell have no structural constraints and therefore no such conflict. The third cell contains models with structural constraints but there are no conflict between the syntactic and semantic information. For simplicity and lack of space, we only report on the comparison of the first two cells in this paper. This comparison is also the most interesting and enlightening. 


\subsection{Independent Variable}

The independent variable in this study is the information model and it consists of two levels -- conflicting model versus non-conflicting model. Conflicting model means that the syntactic information and the underlying semantic information depicted by the model are in contradiction. For non-conflicting models, there is no conflict between the syntactic and semantic information because the structural constraint is absent. The subjects in the experiment were randomly assigned to either the conflicting or nonconflicting group. The two groups received exactly the same set of models except for the presence or absence of structural constraints.

\subsection{Dependent Variables}

The dependent variables in this study are the choice of interpretation, confidence level, and perceived familiarity with domain. The choice of interpretation was captured using multiple-choice questions. Subjects were presented with a choice of Must or May (i.e., mandatory or optional) to categorize the information models. These two choices (i.e., Must and May) were randomly ordered to counter a possible order effect. The confidence of interpretation and perceived familiarity with domain have values ranging from 1 to 7, with 1 indicating the lowest and 7 indicating the highest. An example of the question is shown in Appendix A.

\subsection{Task Characteristics}

The study uses familiar problem domains such as university course selection and enrollment. These familiar domains were chosen to allow subjects to use common sense reasoning to help them interpret the information models. The domain characteristics are controlled by giving the two groups the same set of models. The subjects received either 8 conflicting models or 8 non-conflicting models. The models were randomly ordered so that each subject received a uniquely ordered questionnaire. Again, this is to counter any possible order effect. The same training set that describes the basic information modeling constructs (e.g., entity, relationship, structural constraints) and some examples was provided to both groups of subjects. They were asked to read the training set prior to answering the questionnaire. No formal training or discussion was conducted.

The experimental task required the subjects to select the appropriate interpretation (i.e., mandatory or optional) for a set of information models. They were also asked their confidence level and their perceived familiarity with the domain. The use of interpretation task for the study is explained below. 


\subsection{Use of Interpretation Task}

There are two task categories in information modeling -- model interpretation and model construction. Model interpretation involves interpreting or validating the information given in the information model, whereas model construction requires the subjects to construct an information model from a given case description. Interpretation task was selected for this research.

Interpretation task is an important aspect of information modeling for several reasons. Despite the popularity of end-user computing, large scale information models are still been developed by analysts and validated by end-users (Kim 1990). Thus, the ability of the end-users to correctly understand and validate an information model is important to the success of systems development. Secondly, we strongly believe that information models, as a communication vehicle in information modeling, should be end-user driven rather than analyst driven. As professionals, analysts could afford the time and energy to learn the modeling techniques, whereas the same could not be said about end-users. Therefore, modeling constructs and construct representations that facilitate validation by end-users may be more appropriate and important than constructs that directly correspond to subsequent design techniques or programming languages. Thirdly, even for analysts, there is the constant need to review current and old information models for modification and maintenance purposes. In other words, model interpretation is a task that is common to both analysts and end-users, whereas model construction is mainly done by analysts.

Model interpretation is also a task that is reasonably within the range of the analytic tools we have available from behavioral research. It is a task of considerable but manageable complexity. Because of the intrinsic importance of the task itself, and the tractable complexity of the task, studies on information model interpretation are a natural starting point in the behavioral study of information modeling.

\subsection{Experimental Results and Discussions}

\subsection{Subject Characteristics}

A total of 24 subjects participated in this experiment. The number of subjects and the number of observations for each group are summarized as follows:

\begin{tabular}{|c||c|c|c|}
\hline Group & $\begin{array}{c}\text { No. of } \\
\text { Subjects }\end{array}$ & $\begin{array}{c}\text { No. of Questions } \\
\text { Per Subject }\end{array}$ & $\begin{array}{c}\text { No. of } \\
\text { Observations }\end{array}$ \\
\hline \hline Conflicting & 13 & 8 & 104 \\
\hline Non-Conflicting & 11 & 8 & 88 \\
\hline
\end{tabular}


The subjects in this study were MIS graduate students and faculty. The experiment was conducted at one of the weekly MIS workshops. This group of workshop participants was randomly assigned to the two groups. One group received information models with no conflict and the other group received information models with conflicting syntactic and semantic information. The randomization ensures that user characteristics are controlled. A demographic information sheet was completed by each subject prior to the experiment. The subjects' expertise in information modeling are measured using a scale of 1 to 5 (1 -- Totally unfamiliar, 5 -- Very familiar). The following table summarizes their expertise with the modeling technique and constructs:

\begin{tabular}{|c||c|c|}
\hline Technique/Constructs & Mean & Std. Dev. \\
\hline \hline ER Model & 4.17 & 0.92 \\
\hline Entity & 4.33 & 0.70 \\
\hline Relationship & 4.21 & 0.72 \\
\hline Cardinality & 3.67 & 1.31 \\
\hline \hline Average & 4.10 & \multicolumn{1}{|c}{} \\
\hline \multicolumn{2}{|c|}{} &
\end{tabular}

Based on the demographic information given, we consider this group of subjects as modeling experts. They were very comfortable with the various modeling constructs with an average familiarity score greater than 4 (out of a full score of 5). This was not surprising since most of the subjects had extensive training in data modeling techniques from the courses they had taken (or taught). Some of the subjects also had industrial experience.

\subsection{Choice of Interpretation}

For this analysis, we are comparing the choice of interpretation between the conflicting and non-conflicting groups. The responses of the non-conflicting group serve as a "benchmark" for the conflicting group. In other words, the answers from the non-conflicting group allow us to evaluate the semantic information without the interference of structural constraints. These answers can then be compared to the conflicting group. 


\begin{tabular}{|c||c||c|c|c|c|}
\hline & \multicolumn{3}{|c|}{ Conflicting Group } & \multicolumn{2}{c|}{ Non-Conflicting Group } \\
\hline & $\begin{array}{c}\text { Syntactic } \\
\text { Information } \\
\text { Depicted }\end{array}$ & $\begin{array}{c}\text { Number of } \\
\text { Must } \\
\text { Choice }\end{array}$ & $\begin{array}{c}\text { Number of } \\
\text { May } \\
\text { Choice }\end{array}$ & $\begin{array}{c}\text { Number of } \\
\text { Must } \\
\text { Choice }\end{array}$ & $\begin{array}{c}\text { Number of } \\
\text { May } \\
\text { Choice }\end{array}$ \\
\hline \hline Q1 & $(0, *)$ & 1 & 12 & 8 & 3 \\
\hline Q2 & $(1, *)$ & 12 & 1 & 0 & 11 \\
\hline Q3 & $(0, *)$ & 1 & 12 & 4 & 7 \\
\hline Q4 & $(0, *)$ & 2 & 11 & 6 & 5 \\
\hline Q5 & $(0, *)$ & 2 & 11 & 10 & 1 \\
\hline Q6 & $(1, *)$ & 12 & 1 & 0 & 11 \\
\hline Q7 & $(1, *)$ & 13 & 0 & 1 & 10 \\
\hline Q8 & $(0, *)$ & 0 & 13 & 2 & 9 \\
\hline
\end{tabular}

The table above depicts the choice of interpretation for the two groups. The majority's choice for each question is emphasized. There are two interesting observations in the table. First, consider the choices of the conflicting group. Notice that for all of the $\mathbf{8}$ questions, the choices of the majority in this group are consistent with the syntactic information depicted in the information models. For example, Q7 has a structural constraints of $(1, *)$ and all the subjects in the conflicting group selected Must as their choices whereas Q8 has structural constraints of $\left(0,{ }^{*}\right)$ and all the subjects selected May as their choices. Second, most of the majority's choices in the non-conflicting group are the exact opposite of those in the conflicting group. Take Q6 for example; all the subjects in the non-conflicting group selected May as their choices whereas all, except 1, in the conflicting group selected Must as their choices.

The findings, thus, seem to indicate that the syntactic information presented by structural constraints had a strong impact on the way information models were interpreted. When structural constraints were given, almost all the subjects followed the structural constraints depicted and ignored the underlying semantics of the information models. The following diagrams depict the number of May choices and Must choices for the two groups.

From a psychological point of view, these subjects exhibited attentional bias. They attended only to the syntactic information depicted by the structural constraints when they should have been attending to both the syntactic and semantic information. We use the term attentional bias here to mean the failure to consider alternative possibilities or the failure to look for evidence against an initial possibility based on structural constraints. This behavior can also be known as "semantic fallacy"2 or semantic negligence which means that the subjects ignore the underlying semantics depicted by the information models and focus mainly on the structural constraints given, even for models that had obvious contradiction between the structural constraints and the underlying semantics. A discussion with two of the

2 This can be compared to the "base rate fallacy" coined by Tversky and Kahneman (1982) in the decision making literature. 
subjects in the conflicting group after the experiment revealed that they simply looked at the structural constraints to determine the interpretation. They did not even bother to look at the wordings for the entities and relationships depicted in the information models.

\subsection{Confidence Level and Perceived Domain Familiarity}

The confidence level and perceived domain familiarity for the two groups were analyzed using General Linear Models (GLM) (SAS 1985). The statistics show that there are significant differences between the conflicting and non-conflicting groups for confidence level and domain familiarity (i.e., $p<0.0001$ and $p<0.0028$ respectively). The results are summarized in the table below (DF -- Degree of Freedom; SS -- Sum of Square; MS -- Mean Square; F -- F Statistic).

\begin{tabular}{|c|c|c|c|c|c|}
\hline Measure & DF & S S & M S & F & Pr $>$ F \\
\hline \hline Confidence Level & 1 & 76.26 & 76.26 & 24.09 & 0.0001 \\
\hline Domain Familiarity & 1 & 21.82 & 21.82 & 9.17 & 0.0028 \\
\hline
\end{tabular}

\section{GLM statistics for Confidence Level and Domain Familiarity}

The means and standard deviations for both group are summarized in the table below. To our surprise, the conflicting group rated confidence level and perceived domain familiarity significantly higher than the non-conflicting group.

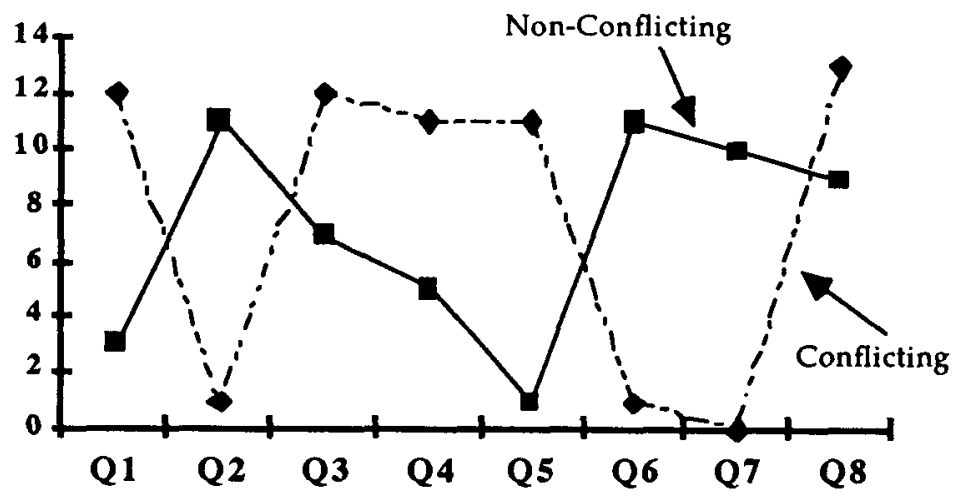

Number of May Choices for the Two Groups 


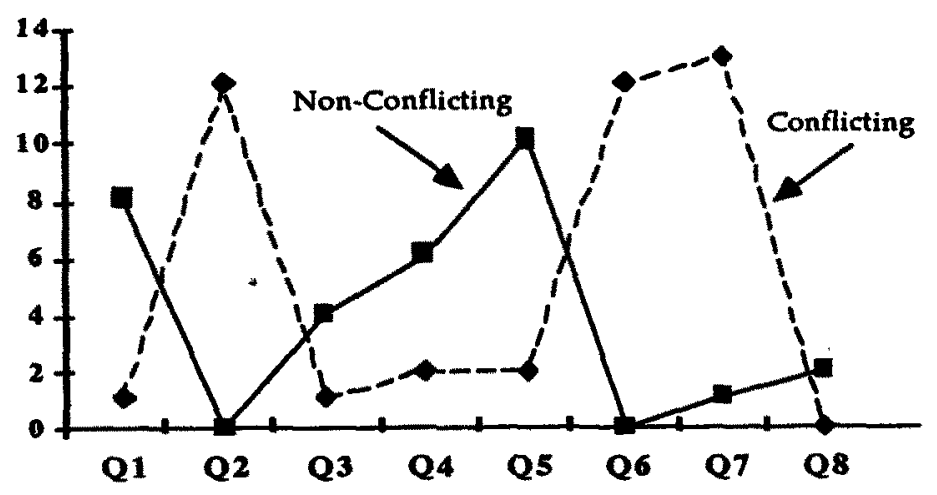

Number of Must Choices for the Two Groups

As can be seen, most of the choices of interpretation for the two groups are directly opposite of one another. The results indicate that those subjects that were not given the structural constraints interpreted the information models based on the underlying semantics. However, subjects who were given the structural constraints (even though they were in conflict with the underlying semantic information), simply followed the structural constraints in their interpretation.

\begin{tabular}{|c||c|c|}
\hline Group & $\begin{array}{c}\text { Confidence Level } \\
\text { Mean (Std. Dev.) }\end{array}$ & $\begin{array}{c}\text { Domain Familiarity } \\
\text { Mean (Std. Dev.) }\end{array}$ \\
\hline \hline Conflicting & $6.23(1.28)$ & $5.78(1.17)$ \\
\hline Non-Conflicting & $4.97(2.23)$ & $5.10(1.89)$ \\
\hline
\end{tabular}

\section{Means and Standard Deviations for the Two Variables}

The results were puzzling. Not only does the existence of syntactic information (i.e., structural constraints) had a significant effect on the interpretation of the information models, but it also increased the subjects confidence and perceived familiarity of the domain. How could these subjects who are trained and experienced in information modeling rate the confidence level and domain familiarity of clearly conflicting information models higher than those that are non-conflicting? To fully understand the puzzle, we analyzed the phenomena at the individual question level. The detailed statistics (i.e., mean and standard deviation) for each question are summarized in the table below. 


\begin{tabular}{|l|c|c|}
\hline & $\begin{array}{c}\text { Confidence } \\
\text { Level } \\
\text { Mean (Std. Dev.) }\end{array}$ & $\begin{array}{c}\text { Domain } \\
\text { Familiarity } \\
\text { Mean (Std. Dev.) }\end{array}$ \\
\hline \hline & \multicolumn{2}{|c|}{ Conflicting } \\
\hline Group \\
\hline Q1 & $6.38(1.12)$ & $6.15(0.80)$ \\
\hline Q2 & $6.38(0.87)$ & $6.46(0.52)$ \\
\hline Q3 & $6.54(0.66)$ & $6.00(0.82)$ \\
\hline Q4 & $6.08(1.66)$ & $6.38(0.77)$ \\
\hline Q5 & $5.92(1.75)$ & $5.85(0.90)$ \\
\hline Q6 & $5.77(1.88)$ & $4.46(1.66)$ \\
\hline Q7 & $6.31(1.11)$ & $5.38(1.33)$ \\
\hline Q8 & $6.46(0.66)$ & $5.54(0.97)$ \\
\hline \hline & Non-Conflicting & Group \\
\hline \hline Q1 & $4.82(2.18)$ & $5.27(1.90)$ \\
\hline Q2 & $5.45(2.46)$ & $5.45(1.92)$ \\
\hline Q3 & $4.91(2.30)$ & $4.91(1.70)$ \\
\hline Q4 & $5.18(2.44)$ & $6.18(1.60)$ \\
\hline Q5 & $5.27(2.37)$ & $5.45(1.86)$ \\
\hline Q6 & $5.00(2.32)$ & $4.18(2.27)$ \\
\hline Q7 & $4.55(2.25)$ & $4.36(1.96)$ \\
\hline Q8 & $4.55(2.07)$ & $5.00(1.67)$ \\
\hline
\end{tabular}

The question by question analysis of the study reveals more surprises. The confidence level for every question in the conflicting group is higher than the corresponding question in the non-conflicting group. Similarly for domain familiarity, all the conflicting questions have a higher perceived domain familiarity than their corresponding non-conflicting questions.

The table also shows strong differences between the two groups in terms of confidence level. The lowest confidence level in the conflicting group (i.e., 5.77) is still higher than the highest confidence level in the non-conflicting group (i.e., 5.45). One probable explanation is that the subjects treated structural constraints as part of the information and felt more comfortable with its presence. This is not surprising as these subjects are all experts in the information modeling technique. It is, therefore, likely that the additional information provided by the structural constraints increased their confidence level. Another possibility is that, with the missing cardinality, the subjects have to retrieve and apply their domain assumptions. This forces them to review and question their domain knowledge, which leads to a lower confidence level. 
The following diagrams depict the confidence level and perceived domain familiarity for each of the questions.

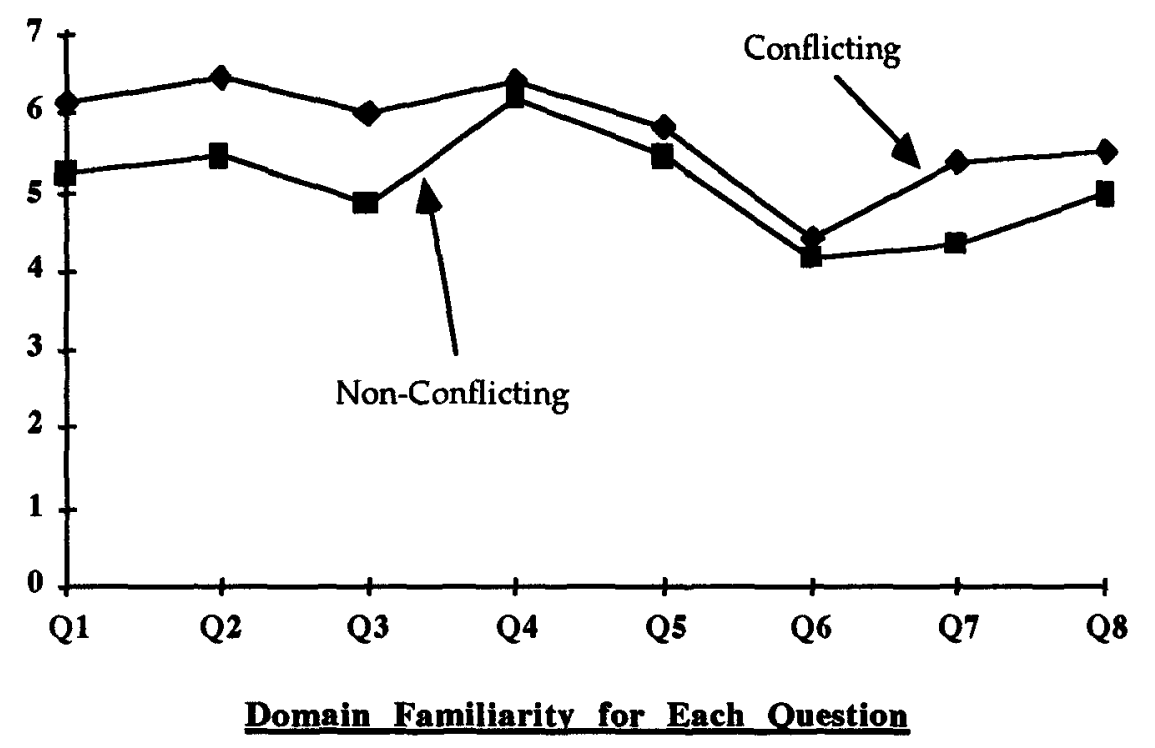

Although the effect on confidence level can be explained by the additional information provided by the structural constraints, the impact on domain familiarity is harder to explain. How could the perceived domain familiarity be higher in the conflicting group? Perceived domain familiarity is determined by the entire information model (i.e., entities, relationship, and structural constraints (if present)) and how closely the information conveyed by the model fit the reality. One possible explanation is that these subjects placed too much faith on the structural constraints. They made up scenarios (however far-stretched that might be) to convince themselves that the structural constraints were correct. Another plausible explanation is that the subjects did not even realize the conflict. This again goes back to the notions of attentional bias and semantic negligence. They looked at the information models but they did not see the conflicts. How could these phenomena be explained from a theoretical perspective?

\subsection{Theoretical Explanation of Results}

Trying to make sense of the results, we reviewed the cognitive psychology literature. The learning paradigm in cognitive psychology provides a plausible theoretical explanation for the results. First, a few words on learning. 
Minsky (1986) defines learning as "an omnibus word for all the processes that lead to long-term changes in our minds." Similarly, Thorpe (1963) defines learning as "that process which manifests itself by adaptive changes in individual behavior as a result of experience," and Wingfield (1979) defines it as "a relatively permanent change in behavior or knowledge brought about by practice or experience."

Despite the proliferation of theories, various attempts by psychologists to divide the process of skill learning into stages have been remarkably similar (Fitts 1964, Fitts \& Posner 1967, Anderson 1983, 1995). The three main stages are: cognitive stage, associative stage, and autonomous stage.

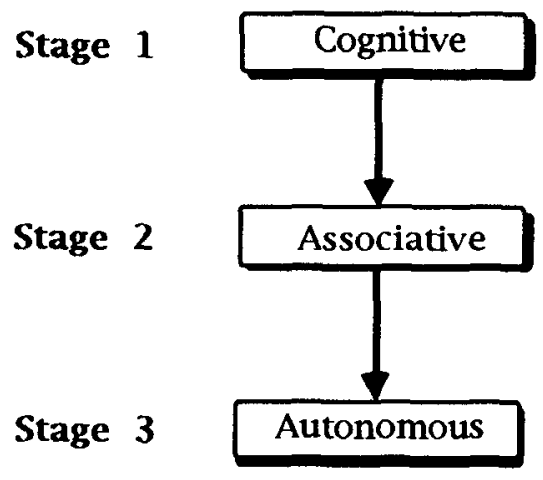

The first phase, cognitive stage, is characterized by discovery of the relevant aspects of the task and the storage of declarative knowledge 3 about the skills. During the associative stage, the skill are chunked, or compiled, into procedural knowledge ${ }^{4}$. In the final or autonomous stage, the procedures undergo a process of continual refinement (i.e., tuning) and strengthening, which results in increased speed and accuracy in performance of the skill. As an example of this transformation, let us take the case of a golfer. A novice golfer, in preparing to hit the ball, may verbalize, "Bend your knees," "Head down," "Keep your left arm straight,", and so on. However, an expert golfer could execute these processes in split seconds and without conscious awareness.

Because the subjects in this experiment are modeling experts, they are most likely to be at the automatic stage in their learning curve for information modeling. At the automatic stage, processes are run without any conscious allocation of attention (Best 1992). Gagne et al. (1993) stated that automated skills are executed almost unconsciously when certain conditions are met. Logan (1988) also stressed that automation is "fast, effortless, autonomous, stereotypic, and unavailable to conscious awareness." Thus, these modeling experts, who are used to interpreting information

3 Declarative knowledge is knowing that something is the case. It is knowledge of facts, theories, events, and objects.

4 Procedural knowledge comprises the skills to perform something. It includes motor skills, cognitive skills, and cognitive strategies. 
models and who have formed highly-specialized modeling-domain-specific strategies, simply attended to the structural constraints depicted in the information models, as they usually do, and ignored the semantic information depicted by the models.

Automaticity of the processes also explains the attentional bias and semantic negligence exhibited by the modeling experts. In other words, those subjects in the conflicting group simply looked at the structural constraints to decide on the Must or May choice and unconsciously ignored the semantic information in the models. Subjects in the non-conflicting group, on the other hand, are exposed to information models that were incomplete (i.e., missing structural constraints). For these information models, the subjects could not activate the automatic skill because certain conditions (i.e., structural constraints) were not met. They were, therefore, forced to evaluate the models based on the semantic information. Thus, differences arose between the two groups.

\subsection{Conclusions}

An individual's use of multiple decision strategies in different situations, including various simplifying methods or choice heuristics, is an adaptive response of a limited-capacity information processor to the demands of complex decision tasks.

-- Payne, Bettman and Johnson, 1993, p. 2

The objective of this research is to understand human cognition in information modeling. The goal is to bridge the gap between behavioral studies and the technical research in information modeling. In this study, we investigated the behavior of modeling experts when given conflicting semantic and syntactic information. The findings of this study indicate that modeling experts are highly biased towards syntactical aspects of the models. When structural constraints were not included in the information model, the subjects based their interpretation on the semantic information. However, when structural constraints were given and presented in a way that contradict the semantic information, almost all of the subjects based their interpretation on the structural constraints depicted and ignored the underlying semantics of the information models. Considering the fact that information model is a communication vehicle during systems analysis, such biases might be detrimental to the information systems projects. The experimental results also show that the existence of structural constraints in the information models significantly increased both the confidence level of the subjects and their perceived familiarity of the domain.

We believe this research has significance implications for both academic and practitioners. The cognitive biases exhibited by the subjects are important issues that need to be addressed. For example, a recent Delphi survey of senior IS executives conducted by Niederman et al. (1991) showed that IS executives were concerned with 
data-related issues. In fact, they listed information architecture and data resource as their top two concerns. Approaches to resolving these issues include education and training of the existing and future IS professionals. Documenting such incidents in text books and training materials serve as important reminder to readers.

Our next experiment will investigate the cognitive differences between modeling experts and modeling novices. With this stream of work, we are not attempting to formulate a complete explanatory model for human behavior in information modeling. We are instead making the point that human cognition is an important dimension in information modeling.

Acknowledgments. The authors would like to thank the anonymous referees for their many valuable comments. Many thanks also to the participants of the MIS Workshop for taking part in the study. This research was supported in part by the Natural Sciences and Engineering, and Humanities and Social Sciences Research Councils of Canada.

\section{References}

1. Anderson, J.R. (1983), The Architecture of Cognition, Cambridge, MA: Harvard University Press.

2. Anderson, J.R. (1995), Learning and Memory: An Integrated Approach, John Wiley \& Sons, 1995.

3. Baron, J. (1988), Thinking and Deciding, Cambridge, Cambridge University Press.

4. Best, J.B. (1992), Cognitive Psychology, Third Edition, West Publishing Company.

5. Boar, B.H. (1984), Application Prototyping: A Requirements Definition Strategy for the 80's, New York, John Wiley \& Sons.

6. Boehm, B.W. (1973), Software and Its Impact: A Quantitative Assessment, Datamation, 19,5 , pp. 48-59.

7. Brooks, F.P. (1987), No Silver Bullet: Essence and Accidents of Software Engineering, IEEE Computer, pp. 10-19.

8. Curtis, B., Krasner, H., and Iscoe, N. (1988), A Field Study of the Software Design Process for Large Systems, Communications of the ACM, Vol. 31, No. 11, pp. 1268-1287.

9. Elmasri, R. and Navathe, S. B. (1994), Fundamentals of Database Systems, Second Edition, Benjamin/Cummings.

10. Fitts, P.M. (1964), Perceptual-motor Skill Learning, in: A.W. Melton (ed.), Categories of Human Learning, New York: Academic Press. 
11. Fitts, P.M., and Posner, M.I. (1967), Human Performance, California: BrooksCole.

12. Gagne, E.D., Yekovich, C.W., and Yekovich, F.R. (1993), The Cognitive Psychology of School Learning, Harper Colins.

13. Gladden, G.R. (1982), Stop the Life Cycle, I Want to Get Off, Software Engineering Notes, Vol. 7, No. 2.

14. Hogarth, R.M. (1980), Judgment and Choice: The Psychology of Decision. Chichester: John Wiley \& Sons.

15. Holland, J.H., Holyoak, K.J., Nisbett, R.E., and Thagard, P.R. (1986), Induction: Processes of Inference, Learning, and Discovery, MTT Press.

16. Holtzblott, K., and Beyer, H.R. (1995), Requirement Gathering: The Human Factor, Communications of the ACM, Vol. 38, No. 5, pp. 31-32.

17. Kangassalo, H. (1990), Foundations of Conceptual Modeling: A Theory Construction View, In: Information Modeling and Knowledge Bases, Amsterdam, IOS Press, pp. 20-29.

18. Kim, Y.G. (1990), Effects of Conceptual Data Modeling Formalisms on User Validation and Analyst Modeling of Information Requirements, Unpublished Dissertation, University of Minnesota.

19. Kung, C.H, and Solvberg, A. (1986), Activity Modeling and Behavior Modeling, in: Information Systems Design Methodologies: Improving the Practice, Olle T W, Sol H G and Verrijn-Stuart (eds.), Amsterdam: Elsevier Science Publishers B.V. (North-Holland), pp. 145-172.

20. Lientz, B.P., and Swanson, E.B. (1980), Software Maintenance Management, Addison-Wesley. Reading, Mass.

21. Logan, G.D. (1988), Toward an Instance Theory of Automatization, Psychological Review, Vol. 95, No. 4, pp. 492-527.

22. Mayer, R.E. (1983), Thinking, Problem Solving, Cognition, Freeman and Company.

23. Minsky, M. (1986), The Society of Mind, Simon \& Schuster.

24. Mylopoulos, J. (1992), Conceptual Modeling and Telos, in: P. Loucopoulos and R. Zicari (Eds.), Conceptual Modeling, Databases and Case, New York, John Wiley \& Sons, pp. 49-68.

25. Newell, A., and Simon, H.A. (1972), Human Problem Solving, Englewood Cliffs, New Jersey: Prentice Hall.

26. Niederman, F., Brancheau, J.C., and Wetherbe, J.C. (1991), Information Systems Management Issues for the 1990's, MIS Quarterly, pp. 475-500.

27. Payne, J.W., Bettman, J.R., and Johnson, E.J (1993), The Adaptive Decision Maker, Cambridge: Cambridge University Press.

28. Ramamoorthy, C.V., Prakash, A., Tsai, W., and Usuda, Y. (1984), Software Engineering: Problems and Perspectives, Computer, Vol. 17, No. 10, pp. 191209.

29. Rolland, C., and Cauvet, C. (1992), Trends and Perspectives in Conceptual Modeling, In: P. Loucopoulos and R. Zicari (Eds.), Conceptual Modeling, Databases and Case, New York, John Wiley \& Sons, pp. 27-32.

30. SAS (1985), SAS User's Guide: Statistics, Version 5, SAS Institute Inc. 
31. Siau, K., Wand, Y., Benbasat, I., A Psychological Study on the Use of Relationship Concept -- Some Preliminary Findings, in: Iivari, J., Lyytinen, K., Rossi, M. (eds.), Lecture Notes in Computer Science, Vol. 932, Springer-Verlag, pp. 341-354.

32. Stacy, W. (1995), Cognition and Software Development, Communications of the ACM, Vol. 38, No. 6, pp. 31.

33. Stacy, W., and MacMillian, J. (1995), Cognitive Bias in Software Engineering, Communications of the ACM, Vol. 38, No. 6, pp. 57-74.

34. Thorpe, W.H., (1963), Learning and Instinct in Animals, Second Edition, London: Methuen.

35. Tversky, A. and Kahneman, D. (1982), Judgment Under Uncertainty: Heuristic and Biases, in: Judgment under Uncertainty, Cambridge University Press, pp. 320.

36. Willumsen, G. (1993), Conceptual Modeling in IS Engineering, In: Executable Conceptual Models in Information Systems Engineering, Trondheim, pp. 11-21.

37. Wingfield, A. (1979), Human Learning and Memory: An Introduction, New York: Harper \& Row. 


\section{Appendix A}

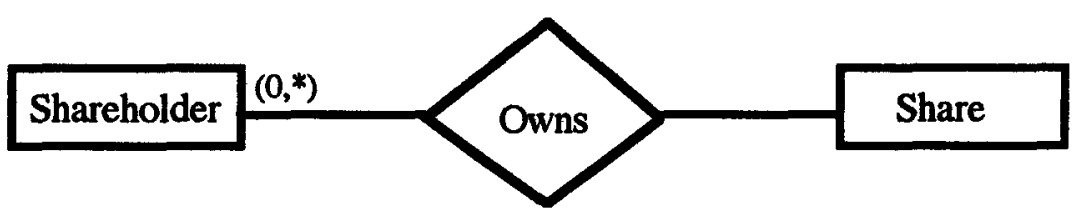

Circle one of the following two options that more correctly reflects the participation of the Shareholder entity type in the Owns relationship type:

1. may own

2. must own

What is your confidence level in the above choice?

$\begin{array}{lllllllll}\text { No confidence } & 1 & 2 & 3 & 4 & 5 & 6 & 7 & \text { Absolute confidence }\end{array}$

What is your familiarity with the domain depicted in the diagram?

$\begin{array}{lllllllll}\text { Not familiar at all } & 1 & 2 & 3 & 4 & 5 & 6 & 7 & \text { Very familiar }\end{array}$ 